\title{
The adhesion molecule cadherin 11 is essential for acquisition of normal hearing ability through middle ear development in the mouse
}

\author{
Yuji Kiyama $^{1} \cdot$ Yayoi S. Kikkawa ${ }^{2} \cdot$ Makoto Kinoshita $^{2} \cdot$ Yu Matsumoto ${ }^{2,3} \cdot$ Kenji Kondo $^{2} \cdot$ Chisato Fujimoto $^{2} \cdot$ \\ Shinichi Iwasaki ${ }^{2} \cdot$ Tatsuya Yamasoba $^{2} \cdot$ Toshiya Manabe $^{1}$
}

Received: 6 October 2017 / Revised: 7 April 2018 / Accepted: 24 April 2018 / Published online: 2 July 2018

(c) United States \& Canadian Academy of Pathology 2018

\begin{abstract}
Cadherin 11 (Cdh11), a member of the cadherin adhesion molecule family, is expressed in various regions of the brain as well as the head and ear. To gain further insights into the roles of Cdh11 in the development of the ear, we performed behavioral tests using Cdh11 knockout (KO) mice. KO mice showed reduced acoustic startle responses and increased thresholds for auditory brainstem responses, indicating moderate hearing loss. The auditory bulla volume and ratio of airfilled to non-air-filled space in the middle ear cavity were reduced in $\mathrm{KO}$ mice, potentially causing conductive hearing loss. Furthermore, residual mesenchymal and inflammatory cells were observed in the middle ear cavity of KO mice. Cdh11 was expressed in developing mesenchymal cells just before the start of cavitation, indicating that Cdh11 may be directly involved in middle ear cavitation. Since the auditory bulla is derived from the neural crest, the regulation of neural crest-derived cells by Cdh11 may be responsible for structural development. This mutant mouse may be a promising animal model for elucidating the causes of conductive hearing loss and otitis media.
\end{abstract}

\section{Introduction}

Cadherin-mediated cell-cell adhesion is a fundamental mechanism involved in cell fate specification, tissue organization and morphogenesis during embryonic development [1]. A member of the cadherin family, cadherin 11

Electronic supplementary material The online version of this article (https://doi.org/10.1038/s41374-018-0083-y) contains supplementary material, which is available to authorized users.

Tatsuya Yamasoba

tyamasoba-tky@umin.ac.jp

$\bowtie$ Toshiya Manabe

tmanabe-tky@umin.ac.jp

1 Division of Neuronal Network, Department of Basic Medical Sciences, Institute of Medical Science, University of Tokyo, 4-6-1 Shirokanedai, Minato-ku, Tokyo 108-8639, Japan

2 Department of Otorhinolaryngology, Faculty of Medicine, University of Tokyo, 7-3-1 Hongo, Bunkyo-ku, Tokyo 113-8655, Japan

3 Department of Materials Engineering, Center for Disease Biology and Integrative Medicine, Faculty of Medicine, University of Tokyo, 7-3-1 Hongo, Bunkyo-ku, Tokyo 113-8656, Japan
(Cdh11), is expressed in the mesenchymal cells of the mouse embryo [2]. This expression pattern of Cdh11 differs from that of other cadherins, suggesting a morphogenetic role in mesenchymal development [3]. Cdh11 is also expressed in various regions of the central nervous system $[4,5]$. Mice lacking Cdh11 showed the enhancement of hippocampal long-term potentiation (LTP) without changes in basal synaptic properties [6]. Cdh11-deficient mice also exhibited abnormal performance in acoustic startle response (ASR) and elevated-plus maze tests, suggesting that Cdh11 is involved in anxiety responses [6]. Although these anxiety-related behavioral tests are robust and work well in rodents [7, 8], other physiological factors may influence the results of these tests. For instance, reduced ASRs can be explained by sensorimotor deficits. Indeed, some members of the cadherin family are expressed in the chick cochlea [9], and cadherin 23 and protocadherin 15 are components of the tip link in hair-cell stereocilia [10, 11]. Although it is not well known about Cdh11 expression in the hearing organ of mammal, Cdh11 expression in mesenchymal cells may play a critical role in ear morphogenesis and hence normal hearing ability. To detect the cause of behavioral abnormalities in Cdh11 knockout (KO) mice with a highly homogeneous genetic background, we 
subjected mutant mice to morphological analyses and a series of behavioral and hearing tests examining sensorimotor functions, emotional behaviors and learning abilities. We found that Cdh11 (KO) mice exhibited moderate conductive hearing loss, which may cause impaired ASRs as well as otitis media.

\section{Materials and methods}

\section{Experimental animals}

Cdh11 KO mice were generated as described previously [12]. Homozygous KO and their wild-type (WT) littermates were produced by crossing heterozygous male and female mutant mice, which were backcrossed to $\mathrm{C} 57 \mathrm{BL} / 6 \mathrm{~J}$ mice for eight generations. Three to four mice were housed per cage in a room with a 12-h light/dark cycle (lights on at 7:00 a.m.) and ad libitum access to food and water. This research was approved by the Animal Care and Experimentation Committee of University of Tokyo, and all experiments were performed according to the guidelines provided by the Committee and the National Institute of Health (NIH). For this study, we used the minimum number of mice required to obtain reliable data and tried to minimize their suffering as much as possible.

\section{Behavioral experiments}

Behavioral tests were performed using male mice aged 10-14 weeks and weighing $>15 \mathrm{~g}$ at the beginning of testing. All behavioral tests, except for the startle response elicited by acoustic, air-puff, or electrical stimuli, were conducted in the following sequence: Sensorimotor reflex, wire-hang, open-field, light-dark transition, elevated plusmaze, ASRs/prepulse inhibition (PPI), tail-flick, and contextual and auditory fear conditioning tests. Each test was separated by an interval of at least $36 \mathrm{~h}$. KO mice and their WT littermates were genotyped at the age of 3 weeks, and then used for the tasks. The startle reflex measurement system (SM-100, Kinder Scientific, Poway, CA, USA) was used for assessing ASRs and PPI as described previously [13]. Startle responses elicited by sounds, air-puffs, or electrical shocks were recorded as in the ASR and PPI experiments described above, except that the option for tactile responses (SMTAC, Kinder Scientific) or the electric shock generator (SMSCK, Kinder Scientific) was connected to the startle reflex measurement system. Three types of stimuli (i.e., $110 \mathrm{~dB}$ white noise, 50 pounds per square inch [psi] air-puffs, and $0.3 \mathrm{~mA}$ electric shocks) were presented to elicit startle responses. The methods for other behavioral tasks are described in the Supplementary Methods section.

\section{Assessment of hearing function}

Hearing function was assessed by ABR measurements. $\mathrm{ABR}$ measurements were performed as described previously [14] with minor alterations. Briefly, ABRs following burst tone stimuli $(2,4,8,16,24$ and $32 \mathrm{kHz})$ were measured. Before ABR experiments, male mice were anaesthetized via intramuscular injection with a mixture of xylazine hydrochloride $(10 \mathrm{mg} / \mathrm{kg})$ and ketamine hydrochloride $(40 \mathrm{mg} / \mathrm{kg})$, and recording electrodes were placed. For differential voltage recordings, two recording needle electrodes were placed subcutaneously at the vertex and below the test ear, and a ground needle electrode was positioned below the contralateral ear. Sound stimulation of 15-ms duration and 1-ms rising and falling time was applied at $11 \mathrm{~Hz}$. Responses of 1024 sweeps were averaged at each intensity level (5-dB steps) to assess the threshold, which was blindly determined as the lowest intensity level at which a clear, reproducible waveform was visible in the trace.

\section{Morphological analysis}

Micro-CT and histological examinations were performed to analyze middle and inner ear morphology. After ABR testing, a subset of mice killed by deep halothane anesthesia was scanned using the micro-computerized tomography (micro-CT) system (InspeXio SMX-90CT: Shimadzu, Kyoto, Japan). The data were analyzed using the MacBiophotonics ImageJ program for three-dimensional reconstruction, distance measurement, and volumetric analysis. A detailed volumetric analysis protocol is shown in Supplementary Figure 2. A subset of mice scanned with micro-CT was reused for histological analysis, in which dissected tissues were fixed in $4 \%$ paraformaldehyde at $4{ }^{\circ} \mathrm{C}$, dehydrated through a series of ethanol washes, embedded in paraffin wax, sectioned into $5-\mu \mathrm{m}$ thick slices, and stained.

\section{In situ hybridization}

As described previously [2], a PstI-SmaI fragment of mouse Cdh11 was subcloned into transcription vector PSPT18 and labeled with digoxigenin-labeled UTP using the SP6/T7 transcription kit (Roche Diagnostics, Mannheim, Germany). Embryonic day 16.5 (E16.5) embryos were obtained from the uterus of pregnant $\mathrm{C} 57 \mathrm{BL} / 6 \mathrm{~J}$ mice anaesthetized via intraperitoneal injection with 2,2,2-tribromoethanol (250 $\mathrm{mg} / \mathrm{kg}$ body weight). Dissected heads from embryos anesthetized on ice were washed with phosphate-buffered saline (PBS) and frozen in optimal cutting temperature (OCT) compounds (Sakura, Finetek, Tokyo, Japan). Sagittal sections with a thickness of $10 \mu \mathrm{m}$ were stored at $-80^{\circ} \mathrm{C}$ for 
Fig. 1 Measurements of acoustic startle responses (ASRs) and prepulse inhibition (PPI). a ASRs elicited at various sound levels. Following the presentation of the sounds with five different stimulus intensities $(70,90,100,110$, and $120 \mathrm{~dB})$ in wild-type (WT, $n=24)$ and knockout $(\mathrm{KO}, n=18)$ mice, maximum startle responses (mean \pm SEM) were recorded. The level of the background noise was $70 \mathrm{~dB}$. b Percentage of PPI of ASRs recorded during the ASR measurement sessions shown in a. Two acoustic prepulses (75 and $80 \mathrm{~dB}$ ) were presented $100 \mathrm{~ms}$ before the onset of ASR sound stimulation. The percentage of PPI of ASRs measured with each prepulse stimulus (mean \pm SEM) is shown. c A scatter-plot diagram showing the correlation between the maximum startle amplitude and time at which the maximum startle amplitude was recorded for all ASR trials with the 120$\mathrm{dB}$ stimulus shown in $\mathbf{a}$. $\mathbf{d}$

Startle responses of WT $(n=6)$ and $\mathrm{KO}(n=6)$ mice to acoustic (110 dB, $40 \mathrm{~ms}$ ), tactile (50-psi air-puffs) and electric shock (0.3 $\mathrm{mA})$ stimuli. The asterisks denote statistically significant differences $(* P<0.05, * * P<$ 0.01 , and $* * * P<0.001)$ tested by Student's $t$-test. a

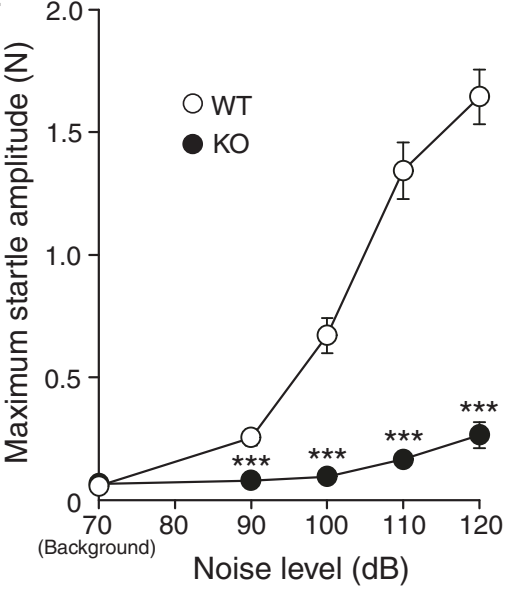

C

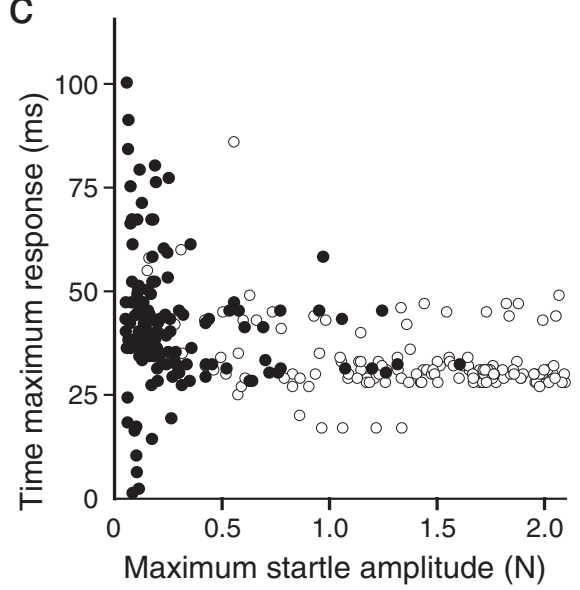

b
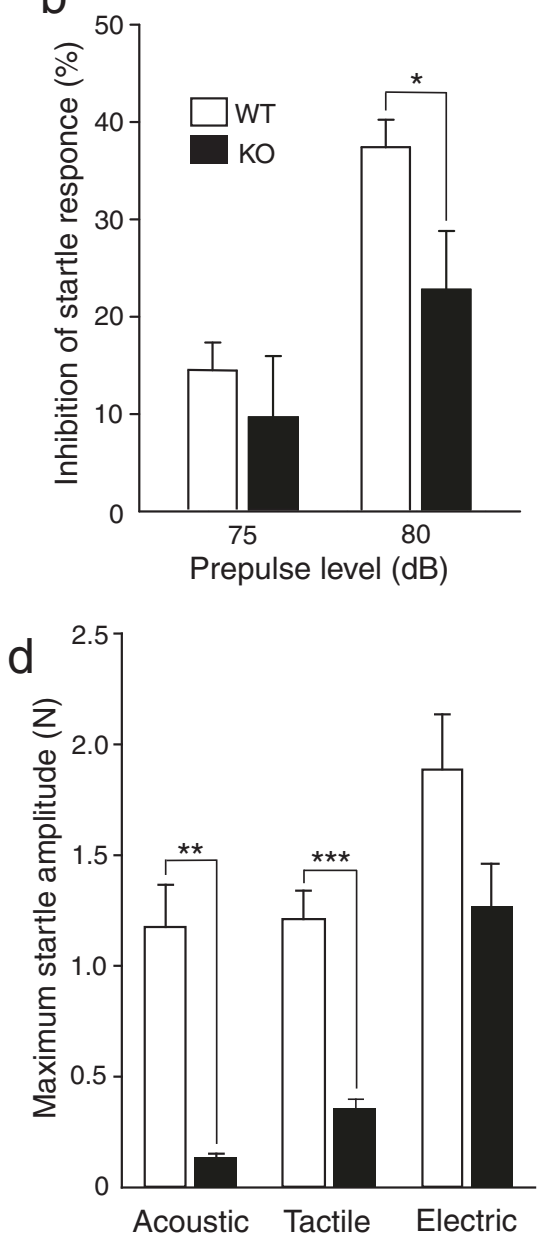

later analysis. For in situ hybridization, slides were fixed with 4\% paraformaldehyde in PBS. After 10 min of acetylation with $0.25 \%$ acetic anhydrate in $0.1 \mathrm{M} \mathrm{HCl}(\mathrm{pH} 8.0)$, prehybridization was carried out in hybridization solution (50\% formamide, $5 \times$ standard saline citrate (SSC), $250 \mu \mathrm{g} /$ $\mathrm{ml}$ yeast tRNA, $5 \times$ Denhardt's solution and $500 \mu \mathrm{g} / \mathrm{ml}$ salmon sperm DNA in diethylpyrocarbonate-treated $\mathrm{H}_{2} \mathrm{O}$ ) at room temperature for $1 \mathrm{~h}$. Slides were hybridized with 300 $\mathrm{ng} / \mathrm{ml}$ of riboprobes in the hybridization solution at $72{ }^{\circ} \mathrm{C}$ overnight. Sections were washed three times in $0.2 \times \mathrm{SSC}$ at $72{ }^{\circ} \mathrm{C}$ for $30 \mathrm{~min}$, and then blocked in $1 \%$ blocking reagent (GE Healthcare, Tokyo, Japan). The tissue sections were exposed to anti-digoxigenin-AP Fab fragments (Roche Diagnostics) at a concentration of 1:1000 in a 1\% blocking reagent overnight at $4{ }^{\circ} \mathrm{C}$. Slides were washed and placed in nitro-blue tetrazolium chloride/5-bromo-4-chloro-3'-indolyphosphate p-toluidine salt solution (Roche Diagnostics) until a blue color appeared.

\section{Immunohistochemistry}

E16.5, E18.5 embryos were obtained from the uterus of pregnant C57BL/6J mice anaesthetized via intraperitoneal injection with 2,2,2-tribromoethanol $(250 \mathrm{mg} / \mathrm{kg}$ body weight). Dissected cranial bases from E16.5, E18.5 and postnatal day $2(\mathrm{P} 2)$ embryos anesthetized on ice were fixed in $4 \%$ paraformaldehyde in PBS overnight at $4{ }^{\circ} \mathrm{C}$. Tissues were transferred to 15 and $30 \%$ sucrose in PBS for several hours. Tissues in $30 \%$ sucrose and OCT compound mixture (1:1) were frozen and stored at $-80^{\circ} \mathrm{C}$. Sagittal sections with a thickness of $20 \mu \mathrm{m}$ were used. Rabbit polyclonal anti-cadherin 11 antibody (1:100; Thermo Fisher, 71-7600) was used for primary antibody. The biotin-streptavidin (BSA) staining method with the Histofine immunostaining kit (Nichirei, Tokyo Japan) and 3,3'-diaminobenzidine, tetrahydrochloride (DAB) tablet (WAKO, Osaka Japan) was utilized for detection. 


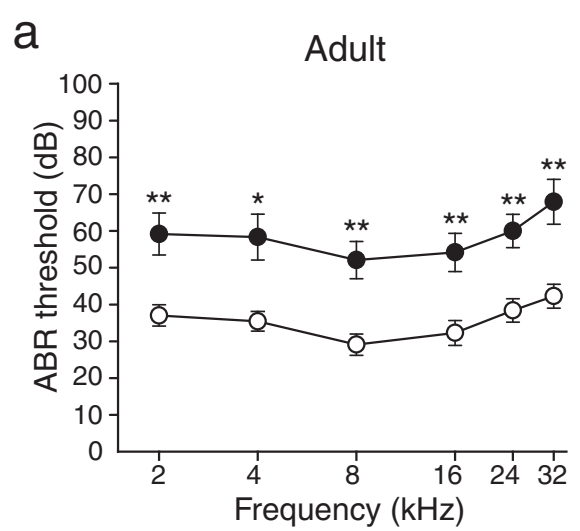

Fig. 2 Measurements of auditory brainstem response (ABR) and distortion product otoacoustic emission (DPOAE). a, b The ABR thresholds (mean $\pm \mathrm{SEM}$ ) of 2-week-old (a: wild-type [WT], $n=6$; knockout $[\mathrm{KO}], n=9$ ) and adult (7-12 weeks old: b: WT, $n=11$; KO,

\section{Statistical analysis}

Comparisons between genotypes were made by Student's $t$ test and $X^{2}$ analysis $* P<0.05, \quad * * P<0.01$, and $* * * P<0.001$.

\section{Results}

\section{Behavioral characteristics of Cdh11 KO mice}

It is well established that genetic background significantly affects mice behavior. Among mouse strains, the C57BL/ 6 strain performs well in hippocampus-dependent learning and anxiety-related tasks and shows standard ASR amplitudes [15]. Since our KO mice were produced by homologous recombination in CJ7 ES cells derived from the $129 \mathrm{SV} / \mathrm{Sv}$ strain [12], we backcrossed $\mathrm{KO}$ mice to the C57BL/6 strain for eight generations to more precisely examine the effect of the Cdh11 disruption. Though KO mice were viable, the number of surviving $\mathrm{KO}$ mice at $\mathrm{P} 21$ was significantly fewer than expected from the number of their wild-type littermates (28\% of expected survival, $P<0.01, X^{2}$ analysis; Supplementary Table 1). This high lethality was similar to that in a previous study using the same allele [16], We subjected survived KO mice to a series of behavioral tests examining sensorimotor functions, emotional behaviors and learning abilities. Behavioral characteristics of KO mice, excluding ASRs and PPI, are shown in Supplementary Figure 1. It is important to note that during the elevated plus-maze test, $\mathrm{KO}$ mice stayed on the open arms longer and entered them more often than WT (Student's $t$ test: Supplementary Figure 1a-c). Contextdependent freezing in $\mathrm{KO}$ mice was significantly enhanced compared to WT mice (Student's $t$ test: Supplementary b

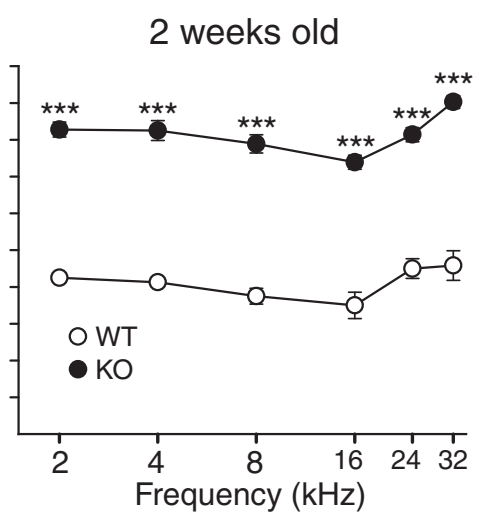

$n=6)$ mice at various sound frequencies $(2,4,8,16,24$, and $32 \mathrm{kHz})$. The asterisks denote statistically significant differences tested by repeated measures two-way ANOVA with the post-hoc Bonferroni test

Figure $1 \mathrm{f}-\mathrm{h})$. However, there was no significant difference between KO and WT mice in the light-dark transition test (Student's $t$ test: Supplementary Figure 1d and e).

Consistent with our previous report [6], the ASRs of KO mice were extremely reduced at various sound levels. KO mice showed significantly reduced startle amplitudes as long as noise level is higher than $70 \mathrm{~dB}$ of background noise $(P<0.001$, Student's $t$ test: Fig. 1a). For the 75-dB prepulse, there was no significant difference between genotypes ( $P=0.49$, Student's $t$ test) in the extent of PPI (Fig. 1b). In contrast, for the $80-\mathrm{dB}$ prepulse, KO mice showed significantly reduced PPI compared to their WT littermates ( $P=0.04$, Student's $t$ test: Fig. $1 \mathrm{~b}$ ). Since the primary neural circuit mediating the ASR is a simple oligosynaptic pathway, startle response latency is less than $100 \mathrm{~ms}$ [17]. Under our experimental conditions, the latencies to maximum startle amplitude in well-responding WT mice typically ranged from 28 to $50 \mathrm{~ms}$ (Fig. 1c, open circles). The majority of $\mathrm{KO}$ mice did not respond well to most trials. However, when they responded well, their latencies to maximum amplitude were similar to those of WT mice (Fig. 1c, filled circles), suggesting that the primary neural pathway for startle responses was intact in KO mice. Startle responses to various sensory stimuli are mediated by a common neural circuit within the lower brainstem and motoneurons [18]. To investigate possible deficits in the neural circuit of perception in KO mice, we examined startle reflexes elicited by air-puffs and electric foot shocks in addition to acoustic stimuli. As with the acoustic stimuli, the amplitude of startle responses elicited by air-puffs was extremely reduced in KO mice $(P<0.001$, Student's $t$ test: Fig. 1d). However, KO mice exhibited normal responses to electrical foot-shocks ( $P=0.079$, Student's $t$ test: Fig. 1d). These results suggest that, in KO mice, the motor system that elicited startle responses was normal, reduced ASRs 

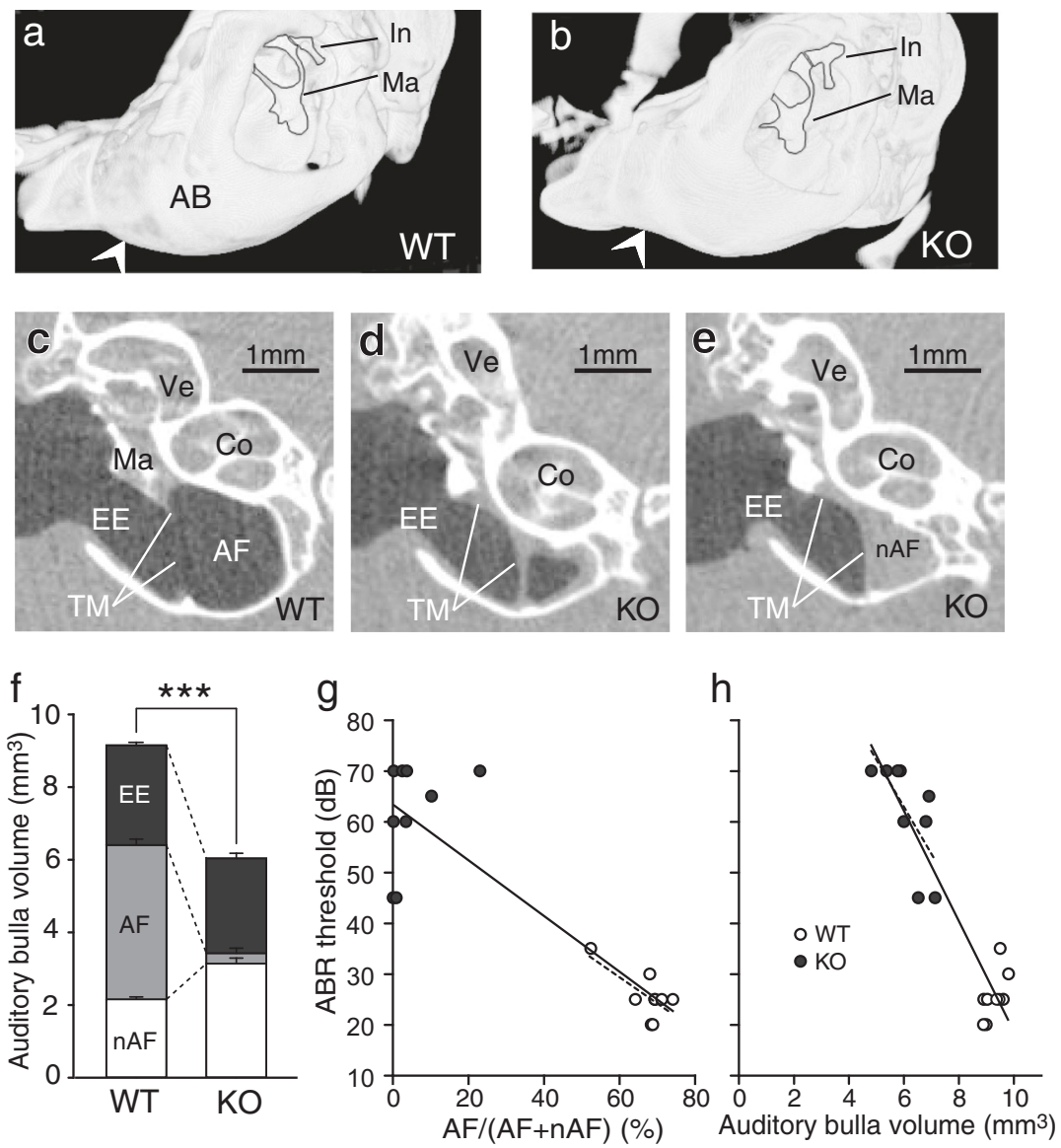

Fig. 3 Micro-computerized tomography (micro-CT) images of the ear and relationship between the auditory brainstem response (ABR) threshold and auditory bulla volume. a, b Three-dimensional reconstructions of the auditory bullae of adult (7-weeks old) wild-type (WT: a) and knockout (KO: b) mice. c-e Representative coronal 2D sections constructed from stacked micro-CT 2D images. A fully cavitated bulla in a WT mouse c, partially cavitated bulla in a $\mathrm{KO}$ mouse $\mathbf{d}$, and bulla without cavitation in a KO mouse e are shown. f Stacked bar charts of the volume of the external ear (EE), air-filled area of the middle ear cavity (AF), and non-air-filled area of the middle ear cavity (nAF) inside the auditory bulla estimated by volumetric analysis (WT, $n=6$

were caused by hearing deficits, and abnormal air-puff responses may also be associated with hearing loss.

\section{KO mice exhibit moderate hearing loss}

To test hearing function in $\mathrm{KO}$ mice, we measured $\mathrm{ABR}$ thresholds using $2-32 \mathrm{kHz}$ sound stimuli. For all frequencies, the ABR thresholds of adult $\mathrm{KO}$ mice were significantly higher than those of WT mice by $20-30 \mathrm{~dB}$ (Student's $t$ test: Fig. 2a), indicating that moderate hearing loss occurred in adult KO mice. Increased ABR thresholds were also found in 2-week-old KO mice (Student's $t$ test: Fig. 2b), suggesting that hearing loss was not caused by age-related degeneration, instead occurred at an early developmental stage. individuals, 11 ears in total; KO, $n=7$ individuals, 14 ears in total). g, h Scatter-plot diagrams showing the correlation between the volume percentage of the $\mathrm{AF}[\mathrm{AF} /(\mathrm{AF}+\mathrm{nAF})]$ and $\mathrm{ABR}$ threshold (g: WT, $n=9$ ears in total; $\mathrm{KO}, n=9$ ears in total) as well as the auditory bulla volume and ABR threshold (h: WT, $n=10$ ears in total; KO, $n=9$ ears in total). AB auditory bulla, AF air-filled area of the middle ear cavity, Co cochlea, EE external ear, In incus, Ma malleus, nAF nonair-filled area of the middle-ear cavity, TM tympanic membrane, Ve vestibule. The asterisks denote statistically significant differences $(* * * P<0.001)$ tested by Student's $t$-test.

\section{KO mice exhibit reduced auditory bulla volume and a middle ear cavitation defect}

To investigate whether morphological defects contributed to the hearing loss observed in $\mathrm{KO}$ mice, we performed morphological analysis of the middle and inner ears. Three-dimensional reconstruction of micro-CT data showed that the auditory bulla encapsulating the middle ear was atrophied in adult KO mice (Fig. 3a, b, white arrow heads). Micro-CT image of the middle ear of WT mice showed that the air-filled middle ear cavity (AF) was clearly visible as a dark region (Fig. 3c), whereas the airfilled area was extremely diminished (Fig. 3d) or disappeared (Fig. 3e) in KO mice. Moreover, in KO mice, the diminished areas were filled with non-air substances. 
Fig. 4 Analysis of middle ear cavitation. a, b, d, e The hematoxylin-eosin (HE)-stained paraffin sections of the middle ear of adult (7-weeks old) wildtype (WT: a, b) and knockout (KO: d, e) mice. The boxed areas in (a) and (d) are enlarged in (b) and (e), respectively. c, f Micro-computerized tomography (micro-CT) images of identical areas to those in the HE-stained paraffin sections of adult WT (a) and KO (d) mice. g-i HE-stained paraffin sections of P5 KO (g) and WT $(\mathbf{h}, \mathbf{i})$ mice. The boxed area in (h) is enlarged in (i). Co cochlea, IC inflammatory cell, MC middle ear cavity, ME middle ear epithelium lining the auditory bulla, TM tympanic membrane, red asterisk a cavitated area of the middle ear
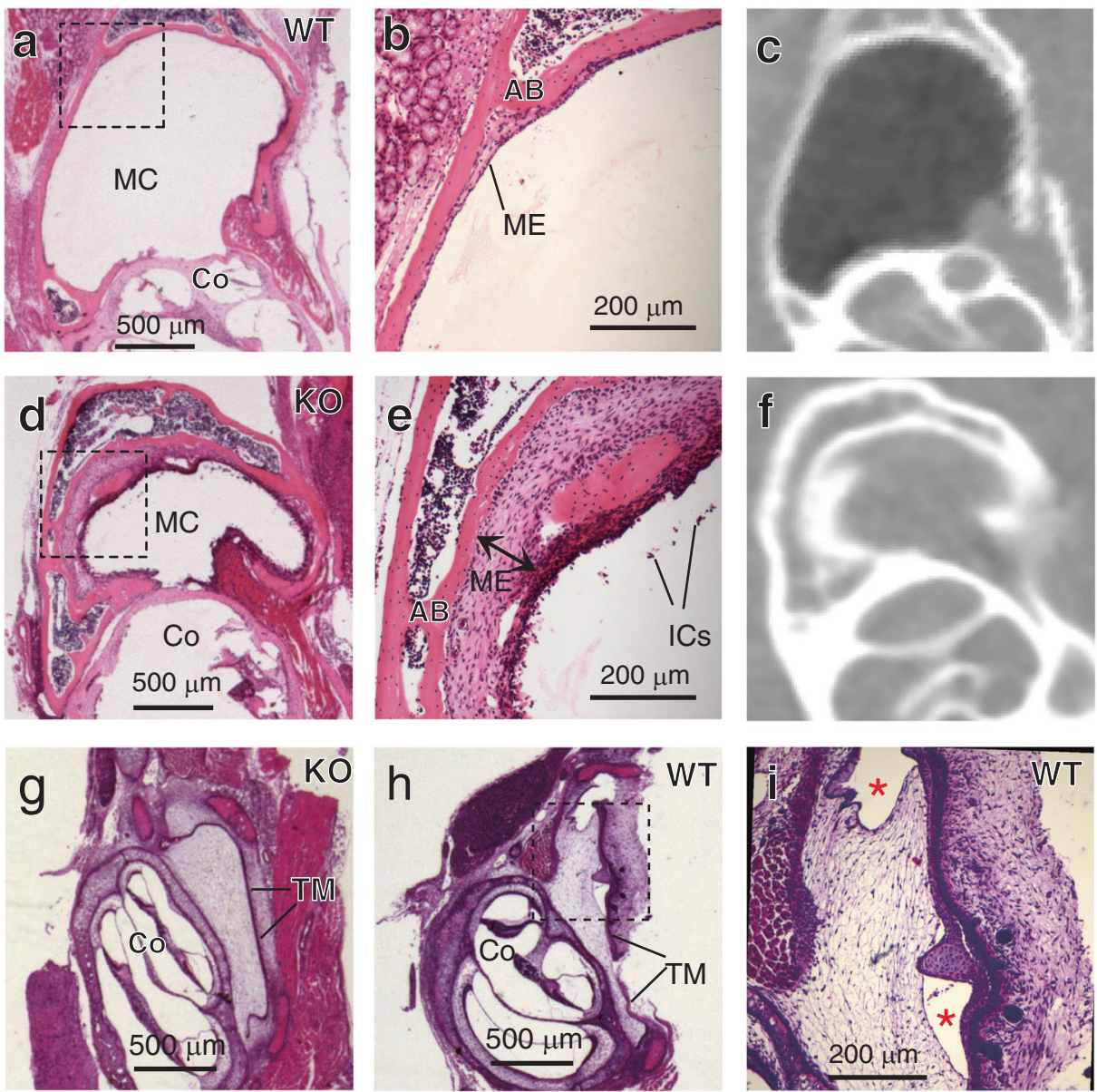

Volumetric analysis with micro-CT (for methods, see Supplementary Figure 2) revealed that the size of the AF of KO mice was significantly smaller than that of their WT littermates $(P<0.001$, Student's $t$ test: Fig. 3f). Furthermore, the size of the non-air-filled middle ear cavity (nAF) was larger in $\mathrm{KO}$ mice compared to WT mice $(P<0.001$, Student's $t$ test: Fig. 3f). The volume of the external ear (EE) was not significantly different between genotypes $(P$ $=0.43$, Student's $t$ test: Fig. 3f). The volume of the auditory bulla (EE, AF, and $\mathrm{nAF}$ ), a region covered by the bone around the middle ear, was statistically smaller in $\mathrm{KO}$ mice compared to WT mice $(P<0.001$, Student's $t$ test: Fig. 3f).

We next examined the possible relationship between hearing impairment and morphological abnormalities of the auditory bulla in KO mice. Scatter-plot graphs of the ABR threshold against the ratio of the air-filled middle ear cavity to the total middle ear cavity $[\mathrm{AF} /(\mathrm{AF}+\mathrm{nAF})]$ indicated a significant correlation in all mice (solid line, $r=-0.895$, $P<0.01$ : Fig. $3 \mathrm{~g}$ ) as well as in WT mice alone (broken line, $r=-0.692, P<0.05$ : Fig. 3g). Moreover, a significant correlation was observed between the $\mathrm{ABR}$ threshold and auditory bulla volume in all mice (solid line, $r=-0.930$, $P<0.01$ : Fig. $3 \mathrm{~h}$ ) as well as in $\mathrm{KO}$ mice alone (broken line, $r=-0.692, P<0.05$ : Fig. $3 \mathrm{~h})$. Thus, it was very likely that the moderate hearing loss observed in $\mathrm{KO}$ mice was caused by impaired cavitation of the middle ear.

The smaller size and loss of cavitation of the auditory bulla in $\mathrm{KO}$ mice were also observed in hematoxylin-eosin (HE)-stained sections during histological analyses (Fig. 4a, d). HE-stained sections were prepared from the same mice that previously underwent micro-CT analysis (Fig. 4c, f). The layer of middle ear epithelial cells lining the auditory bulla of KO mice (ME, two-headed arrow; Fig. 4e) was significantly thicker than that of WT mice (ME; Fig. 4b: WT, $11.8 \pm 0.5 \mu \mathrm{m}, n=4$; KO, $104.0 \pm 19.4 \mu \mathrm{m}, n=6 ; P<$ 0.01 , Student's $t$ test). This indicates that middle ear cavitation, which is caused by the replacement of mesenchymal cells with an air-filled cavity, was not completed in $\mathrm{KO}$ mice. Although there appears to be a cavity inside the epithelial cell layer of KO mouse sections (Fig. 4d, e), this region was not air-filled in most cases, which was similar to micro-CT images of the same area (Fig. 4f) acquired prior to histological sampling. In WT mice sections, the cavity 
Fig. 5 In situ hybridization analysis of cadherin 11 (Cdh11) expression in the middle ear of the embryonic day $16.5(\mathrm{E} 16.5)$ embryo. a, c, e Serial sagittal sections of the middle ear stained by hematoxylin-eosin (HE: a) or hybridized by antisense (c) or sense (e) probes. The boxed areas are enlarged in the right panels $(\mathbf{b}, \mathbf{d}, \mathbf{f})$. AC auditory capsule, Co cochlea, Me mesenchymal cells in the middle ear, TM tympanic membrane, broken lines boundaries between the $\mathrm{AC}$ and $\mathrm{Me}$
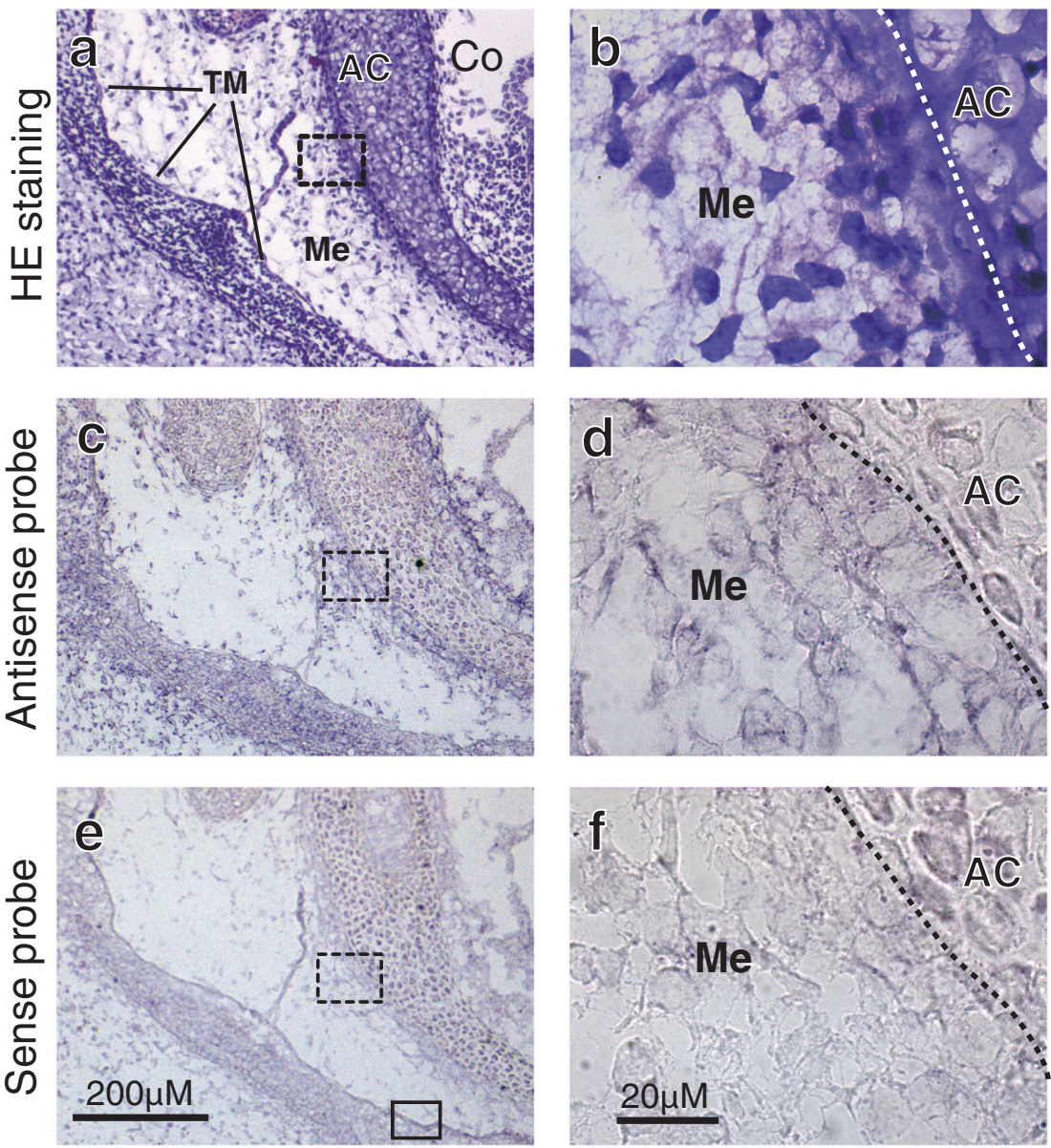

inside the epithelial cell layer was air-filled (Fig. 4a-c). In $\mathrm{KO}$ mice, there were some peculiar cells inside the auditory bulla, which were likely inflammatory cells (ICs: Fig. 4e). Such peculiar cells existed in all middle ear cavities of KO mice we analyzed $(n=6)$. Thus, the middle ear cavity of KO mice appeared to be filled with inflammatory fluid indicative of otitis media. Loss of middle ear cavitation was also found in P5 KO mice (Fig. 4g), whereas partially cavitated air-filled areas were observed in P5 WT mice (red asterisks: Fig. 4h, i).

In contrast to the impairment of middle ear cavitation, the shape and size of adult auditory ossicles, in which the Cdhl1 was not expressed during embryonic stages (Supplementary Figure 3i-q), were not obviously altered (Supplementary Figure 3a-h). Although the Cdhll was expressed in the inner and outer pillar cells of the organ of Corti (Supplementary Figure 4a), inner ear volume (Supplementary Figure $4 \mathrm{~b}$ ) and cochlea shape (Supplementary Figure $4 \mathrm{c}$ and d) were similar between KO and WT mice. Taken together, these results indicate that the moderate hearing loss observed in $\mathrm{KO}$ mice was caused by deficits in middle ear development, and the inner ear may not be involved in the observed hearing loss.

\section{Cdh11 is expressed in middle ear mesenchymal cells}

The previous observations suggested that Cdh11 plays a critical role in auditory bulla development and middle ear cavitation. To examine the role of Cdh11 in these processes, we analyzed Cdh11 expression of in the developing middle ear by in situ hybridization. Since middle ear cavitation starts around birth [19], we analyzed Cdh11 expression of immediately before birth. In theE16.5 embryo, the middle ear cavity was filled with mesenchymal cells (Me: Fig. 5a, b). The sections hybridized with a Cdh11 antisense probe exhibited the Cdh11 mRNA in mesenchymal cells inside the middle ear (Me: Fig. 5c, d). In contrast, staining was hardly detected in the mesenchymal cells of sections hybridized with a sense probe (Me: Fig. 5e, f). These results indicate that Cdh11 is expressed in middle ear mesenchymal cells before birth.

To confirm the expression of Cdh11 in middle ear mesenchymal cells, we re-analyzed immunohistochemically. The sections reacted with anti-cadherin 11 primary antibody exhibited immunostainings in middle ear mesenchymal cells of E16.5 (Me: Fig. 6b, e), E18.5 

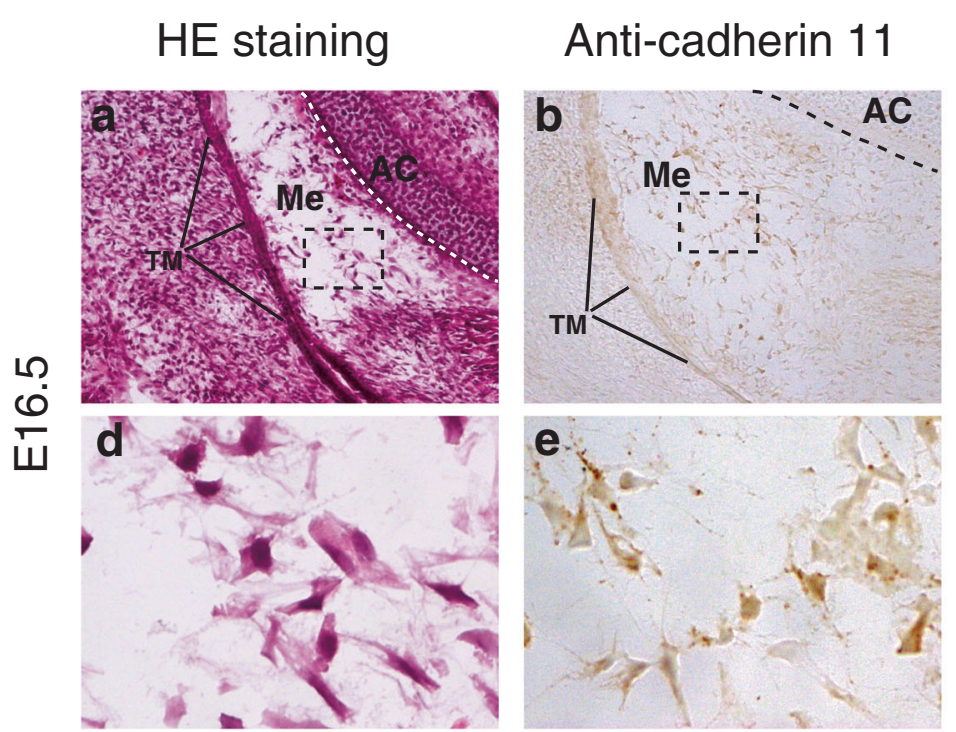

\section{Negative Control}
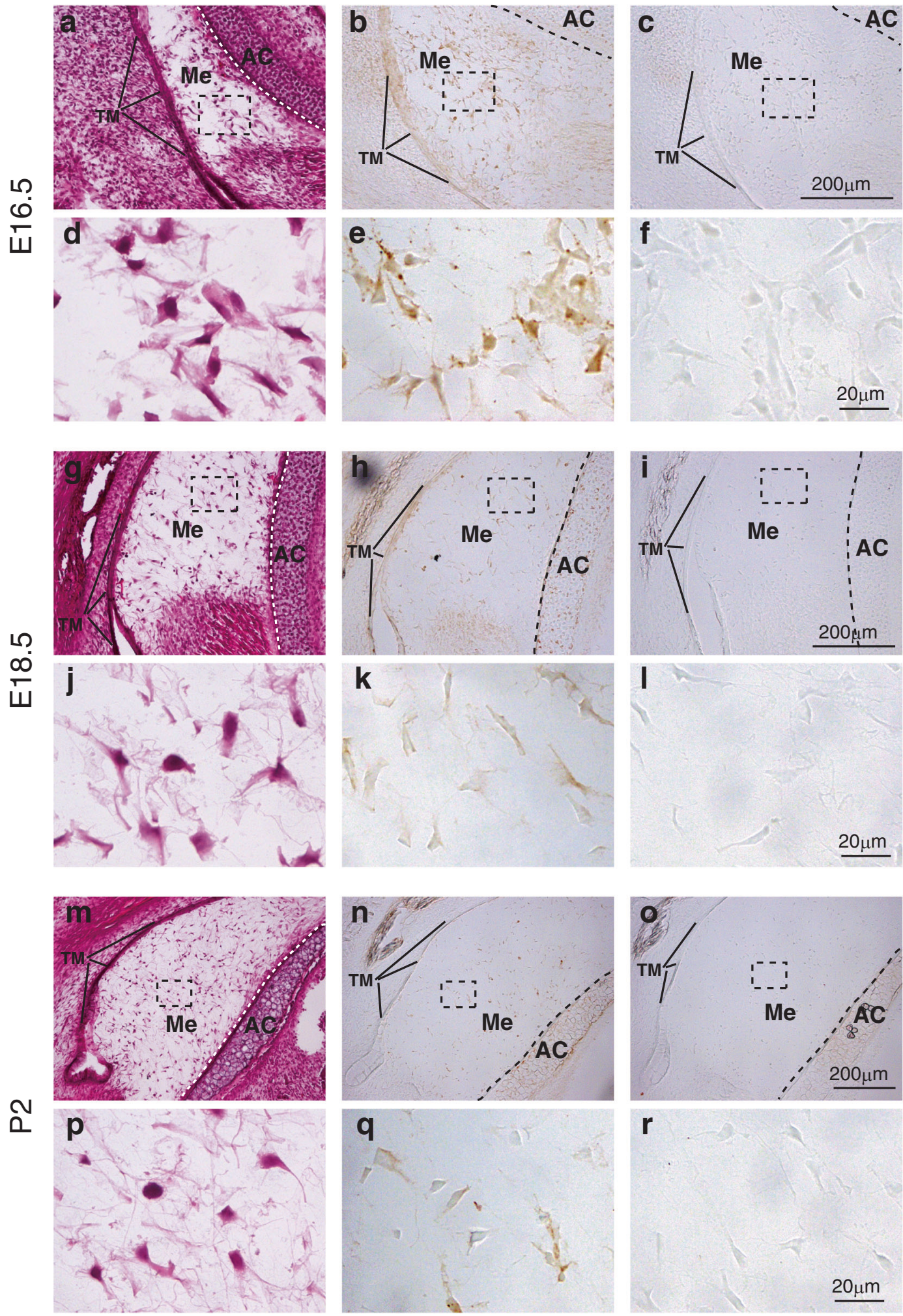

(Me: Fig. 6h, k) and P2 (Me: Fig. 6n, q). On the other hand, negative controls without the primary antibody did not exhibit any stainings (Me: Fig. 6c, f, i, 1, o, r). These results indicate that cadherin 11 protein is also existed in middle ear mesenchymal cells and involved in middle ear cavitation. 
Fig. 6 Immunohistochemical analysis of cadherin 11 protein in middle ear mesenchymal cells of the E 16.5, E18.5 and P2. Sagittal sections of the middle ear stained by hematoxylin-eosin (HE: a, g, m) or stained by anti-cadherin 11 primary antibody $(\mathbf{b}, \mathbf{h}, \mathbf{n})$ or negative control without the primary antibody $(\mathbf{c}, \mathbf{i}, \mathbf{o})$ of E16.5 (a, b, c), E18.5 (g, h, i) and P2 mice ( $\mathbf{m}, \mathbf{n}, \mathbf{o})$. The boxed areas are enlarged in the under panels $(\mathbf{d}-\mathbf{f}, \mathbf{j}-\mathbf{l}, \mathbf{p}-\mathbf{r})$. AC auditory capsule, Co cochlea, Me mesenchymal cells in the middle ear, TM tympanic membrane, broken lines boundaries between the $\mathrm{AC}$ and $\mathrm{Me}$

\section{Discussion}

Using behavioral and morphological analyses, we found that the Cdh11-deficient mouse exhibits a moderate hearing impairment caused by abnormal middle ear development and could be used as a novel animal model of conductive hearing loss (Supplementary Figure 5). Because C57BL/6 mice, to which the mice used in this study were backcrossed, exhibit hearing loss by 200 days of age [20], the hearing loss observed in the young $\mathrm{KO}$ mice from this study is unlikely to be associated with age-related hearing loss. We also found that contextual fear memory was facilitated in KO mice (Supplementary Figure 1f-h). The $C d h 11$ is expressed in limbic system structures such as the hippocampus and amygdala [5, 6], and synaptic plasticity in these brain regions may be associated with fear memory [13, 21]; thus, memory facilitation in $\mathrm{KO}$ mice may be caused by the enhancement of LTP in the hippocampus and/or amygdala [6]. Alternatively, the moderate hearing loss observed in KO mice may have resulted in facilitated memory via some unknown mechanism. Moreover, since hearing loss can influence behavioral characteristics [22, 23], it may be associated with some of the other behavioral abnormalities observed in $\mathrm{KO}$ mice.

To identify the cause of hearing loss in $\mathrm{KO}$ mice, we first searched for abnormalities of the inner ear. Although we found that the $C d h 11$ was expressed in inner and outer pillar cells in the adult cochlea of WT mice (Supplementary Figure 4a), we failed to detect any morphological abnormalities of the inner ear. In contrast, we found clear abnormalities of the middle ear, including smaller- sized auditory bullae and the loss of the AFs (Fig. 3). The loss of the AF cavity likely caused the conductive hearing loss observed in $\mathrm{KO}$ mice. This interpretation is supported by a previous report [24] showing that conductive manipulations, such as partial filling of the middle ear cavity with saline, produced hearing loss accompanied by increases in ABR thresholds. We also found that the size of the auditory bulla was smaller in KO mice; however, it was unclear whether this phenotype was related to hearing loss. We speculate that smaller-sized auditory bullae may interfere with the free movement of the ossicular chain, resulting in hearing loss.
We also observed a thickened epithelium lining the auditory bulla of KO mice (Fig. 4e, f), indicating that residual mesenchymal cells were present and middle ear cavitation was incomplete. The apoptosis of mesenchymal cells [25] and their transformation into epithelial cells [26] may be associated with the formation of middle ear cavitation. Here we showed that the $C d h 11$ was expressed in middle ear mesenchymal cells prior to cavitation (Figs. 5 and 6) and Cdh11-deficient mice exhibited severe cavitation impairment. These findings strongly suggest that Cdh11 plays an essential role in the cavitation of the middle ear. Detailed molecular function of the cadherin 11 on middle ear cavitation is not elucidated. It is possible, however, that the cell adhesion function of cadherin protein between mesenchymal cells have critical rule for middle ear cavitation because disappearing mesenchymal cells during the cavitation are always adhered as a mass $[18,26,27]$. We also observed that KO mice exhibited symptoms of otitis media and residual mesenchymal cells were often present in KO mice. Similar phenotypes were reported in other mutant mice exhibiting otitis media [28, 29]. Moreover, in human infants with congenital anomalies of the ear or other parts of the body, a positive correlation was observed between the amount of mesenchyme remaining in the middle ear and the degree of developmental abnormality of the middle ear [30, 31]. In mammals, endoderm-derived and neural crestderived epithelial cells lining the auditory bulla. Most endoderm-derived epithelial cells have cilia, but neural crest-derived epithelial cells that have underwent a mesenchymal-to-epithelial transformation do not have cilia [26]. It is possible that residual mesenchymal cells seen in KO mice disrupt the composition ratio of ciliated and unciliated epithelium, and therefore increase the infection rate of otitis media. Elucidation of the detailed molecular mechanism of cadherin 11 involved in the formation of middle ear cavitation could shed light on the etiology of otitis media and other middle ear defects.

Middle ear abnormalities have also been observed in other mutant mice [19, 28, 29, 32, 33]. Interestingly, most of these mutant mice exhibited craniofacial dysmorphology similar to that of Cdh11 KO mice as shown in our previous report [6], which is understandable since both the auditory bulla and anterior cranium are derived from neural crest cells $[16,34,35]$. For instance, Tcof1 mutant mice showing severe conductive hearing loss [19] exhibit a deficit in the migration of neural crest cells to the frontonasal prominence and brachial arches, resulting in craniofacial abnormalities [36]. It should be noted that Cdh11 is expressed in neural crest-derived embryonic mesenchymal cells, which constitute the mandibular and maxillary arches that eventually form the jaw [2]. This role of Cdh11 in the formation of the cranium seems to be phylogenetically conserved. Cdh11 is also expressed in the neural 
crest-derived cells of the genus Xenopus [37] and its protrusive activity is essential for craniofacial cartilage formation $[38,39]$. Neural crest-derived cells could also affect the pharyngeal pouches of endoderm that helps form the middle ear by forming tympanic membrane [40, 41]. Thus, lack of Cdh11 in neural crest-derived cells may also influence middle ear formation indirectly. Taken together, the developmental abnormalities of the middle ear and cranium of $\mathrm{KO}$ mice are likely caused by a defect in the migration of Cdh11-expressing neural crest-derived cells. Further studies are needed to understand the precise molecular and cellular mechanisms by which Cdh11 regulates the development of the head.

Acknowledgements We thank Sato S., Numata N., Nagai M., Shiojiri R., Ogawa I., and Miyazawa K. for maintaining mouse colonies, and Watanabe Y., Tsuyuzaki A., Kurasawa Y., Miwa K., Kawahara G. Choi S. and Mori Y. for technical assistance. We are also grateful to Takeichi $\mathrm{M}$. for providing us with $\mathrm{Cdh} 11 \mathrm{KO}$ mice and manuscript comments. We used the SMX-90CT micro-CT scanner at the Center for NanoBio Integration at University of Tokyo, which is supported by the Ministry of Education, Culture, Sports, Science, and Technology (MEXT). This work was supported by Grants-in-Aid for Scientific Research from the Japan Society for the Promotion of Science and the Global Center of Excellence Program "Comprehensive Center of Education and Research for Chemical Biology of the Diseases") from MEXT.

\section{Compliance with ethical standards}

Conflict of interest The authors declare that they have no conflict of interest.

\section{References}

1. Takeichi M. Morphogenetic roles of classic cadherins. Curr Opin Cell Biol. 1995;7:619-27.

2. Kimura Y, Matsunami H, Inoue T, et al. Cadherin-11 expressed in association with mesenchymal morphogenesis in the head, somite, and limb bud of early mouse embryos. Dev Biol. 1995;169347-58.

3. Lee DM, Kiener HP, Agarwal SK, et al. Cadherin-11 in synovial lining formation and pathology in arthritis. Science. 2007;315: 1006-10.

4. Suzuki SC, Inoue T, Kimura Y, et al. Neuronal circuits are subdivided by differential expression of type-II classic cadherins in postnatal mouse brains. Mol Cell Neurosci. 1997;9:433-47.

5. Kimura Y, Matsunami H, Takeichi M. Expression of cadherin-11 delineates boundaries, neuromeres, and nuclei in the developing mouse brain. Dev Dyn. 1996;206:455-62.

6. Manabe $\mathrm{T}$, Togashi $\mathrm{H}$, Uchida $\mathrm{N}$, et al. Loss of cadherin-11 adhesion receptor enhances plastic changes in hippocampal synapses and modifies behavioral responses. Mol Cell Neurosci. 2000;15:534-46.

7. Davis M, Walker DL, Lee Y. Roles of the amygdala and bed nucleus of the stria terminalis in fear and anxiety measured with the acoustic startle reflex. possible relevance to PTSD. Ann N Y Acad Sci. 1997;821:305-31.

8. Hogg S. A. review of the validity and variability of the elevated plus-maze as an animal model of anxiety. Pharmacol Biochem Behav. 1996;54:21-30.
9. Luo J, Wang H, Lin J, et al. Cadherin expression in the developing chicken cochlea. Dev Dyn. 2007;236:2331-7.

10. Siemens J, Lillo C, Dumont RA, et al. Cadherin 23 is a component of the tip link in hair-cell stereocilia. Nature. 2004; 428:950-5.

11. Kazmierczak P, Sakaguchi H, Tokita J, et al. Cadherin 23 and protocadherin 15 interact to form tip-link filaments in sensory hair cells. Nature. 2007;449:87-91.

12. Horikawa K, Radice G, Takeichi M, et al. Adhesive subdivisions intrinsic to the epithelial somites. Dev Biol. 1999;215:182-9.

13. Nakazawa T, Komai S, Watabe AM, et al. NR2B tyrosine phosphorylation modulates fear learning as well as amygdaloid synaptic plasticity. EMBO J. 2006;25:2867-77.

14. Yamasoba T, Schacht J, Shoji F, et al. Attenuation of cochlear damage from noise trauma by an iron chelator, a free radical scavenger and glial cell line-derived neurotrophic factor in vivo. Brain Res. 1999;815:317-25.

15. Crawley JN, Belknap JK, Collins A, et al. Behavioral phenotypes of inbred mouse strains: implications and recommendations for molecular studies. Psychopharmacology. 1997;132:107-24.

16. Bowen CJ, Zhou J, Sung DC, et al. Cadherin-11 coordinates cellular migration and extracellular matrix remodeling during aortic valve maturation. Dev Biol. 2015;407:145-57.

17. Koch M. The neurobiology of startle. Prog Neurobiol. 1999;59: 107-28.

18. Simons-Weidenmaier NS, Weber M, Plappert CF, et al. Synaptic depression and short-term habituation are located in the sensory part of the mammalian startle pathway. BMC Neurosci. 2006;7:38.

19. Richter CA, Amin S, Linden J, et al. Defects in middle ear cavitation cause conductive hearing loss in the Tcof1 mutant mouse. Hum Mol Genet. 2010;19:1551-60.

20. Henry KR, Chole RA. Genotypic differences in behavioral, physiological and anatomical expressions of age-related hearing loss in the laboratory mouse: original papers travaux originaux. Audiology. 1980;19:369-83.

21. Kiyama Y, Manabe T, Sakimura K, et al. Increased thresholds for long-term potentiation and contextual learning in mice lacking the NMDA-type glutamate receptor epsilon1 subunit. J Neurosci. 1998;18:6704-12.

22. Taylor BK, Casto R, Printz MP. Dissociation of tactile and acoustic components in air puff startle. Physiol Behav. 1991;49: 527-32.

23. Vetter DE, Li C, Zhao L, et al. Urocortin-deficient mice show hearing impairment and increased anxiety-like behavior. Nat Genet. 2002;31:363-9.

24. Qin Z, Wood M, Rosowski JJ. Measurement of conductive hearing loss in mice. Hear Res. 2010;263:93-103.

25. Roberts DS, Miller SA. Apoptosis in cavitation of middle ear space. Anat Rec. 1998;251:286-9.

26. Thompson H, Tucker AS. Dual origin of the epithelium of the mammalian middle ear. Science. 2013;339:1453-6.

27. Palva T, Ramsay H. Fate of the mesenchyme in the process of pneumatization. Otol Neurotol. 2002;23:192-9.

28. Yang B, Tian C, Zhang ZG, et al. Sh3pxd2b mice are a model for craniofacial dysmorphology and otitis media. PLoS ONE. 2011;6: e22622.

29. Depreux FF, Darrow K, Conner DA, et al. Eya4-deficient mice are a model for heritable otitis media. J Clin Invest. 2008;118: $651-8$.

30. Takahara T, Sando I, Hashida Y, et al. Mesenchyme remaining in human temporal bones. Otolaryngol Head Neck Surg. 1986;95:349-57.

31. Takahara T, Sando I. Mesenchyme remaining in temporal bones from patients with congenital anomalies. a quantitative histopathologic study. Ann Otol Rhinol Laryngol. 1987;96:333-9. 
32. Rowe TM, Rizzi M, Hirose $\mathrm{K}$, et al. A role of the double-stranded RNA-binding protein PACT in mouse ear development and hearing. Proc Natl Acad Sci USA. 2006;103:5823-8.

33. Wei K, Chen J, Akrami K, et al. Neural crest cell deficiency of cmyc causes skull and hearing defects. Genesis. 2007;45:382-90.

34. McBratney-Owen B, Iseki S, Bamforth SD, et al. Development and tissue origins of the mammalian cranial base. Dev Biol. 2008;322:121-32.

35. Minoux M, Rijli FM. Molecular mechanisms of cranial neural crest cell migration and patterning in craniofacial development. Development. 2010;137:2605-21.

36. Dixon J, Jones NC, Sandell LL, et al. Tcof1/Treacle is required for neural crest cell formation and proliferation deficiencies that cause craniofacial abnormalities. Proc Natl Acad Sci USA. 2006;103: 13403-8.
37. Hadeball B, Borchers A, Wedlich D. Xenopus cadherin-11 (Xcadherin-11) expression requires the $\mathrm{Wg} / \mathrm{Wnt}$ signal. Mech Dev. 1998;72:101-13.

38. Kashef J, Kohler A, Kuriyama S, et al. Cadherin-11 regulates protrusive activity in Xenopus cranial neural crest cells upstream of Trio and the small GTPases. Genes Dev. 2009;23:1393-8.

39. McCusker C, Cousin H, Neuner R, et al. Extracellular cleavage of cadherin-11 by ADAM metalloproteases is essential for Xenopus cranial neural crest cell migration. Mol Biol Cell. 2009;20:78-89.

40. Mallo M. Formation of the outer and middle ear, molecular mechanisms. Curr Top Dev Biol. 2003;57:85-113.

41. Furutera T, Takechi M, Kitazawa T, et al. Differing contributions of the first and second pharyngeal arches to tympanic membrane formation in the mouse and chick. Development. 2017;144: $3315-24$. 